\title{
Histological features of tubercular lymphadenitis in hiv positive patients
}

\begin{abstract}
Tuberculosis is one of the most common causes of lymphadenopathy in HIV positive patients. Though the presenting complaint is same as non HIV patient, the histologic features of lymph node biopsy varies depending on the immune status. The present study is conducted to find out these differences and its relevance in diagnosis.

Material and methods: The histological features seen on lymph node biopsies, done on HIV positive patients who presented with lymphadenopathy, with or without other systemic manifestations over a period of three years were analysed. Seventy four lymph node biopsies were found adequate and provided the material for the present study. The lymph nodes biopsies were fixed in $10 \%$ formalin and were stained using Haematoxylin and eosin (H\&E) stain. Sections were stained for AFB by using ZiehlNeelson method if $\mathrm{H} \&$ E stained slides showed features suggestive of tuberculosis on light microscopy.
\end{abstract}

Results: In the current study, 33 cases (44\%) were diagnosed as tuberculous lymphadenitis and was the second most common cause of lymphadenopathy. The CD4 counts of all these patients ranged from 10-258/ $\mu 1$ with the CD4 count being $<200$ in $84.8 \%$ of cases. In the present study, granulomas were detected in $90.9 \%$ of the cases and were the most common and conspicuous feature. Confluent granulomas were more commonly seen than discrete ones. Most of the cases of caseating $(75 \%)$ had more than $1 \mathrm{AFB} / \mathrm{hpf}$ whereas in the remaining cases (25\%), number was less than $1 / 100 \mathrm{hpf}$. Granulomatous lymphadenitis without caseous necrosis was seen in 2 cases (6.7\%). Microabscess with granular debris without coexisting granuloma was found in 11 cases $(33.3 \%)$ and all showed acid fast bacilli on Ziehl-Neelsen stain. Other features noted in present study were plasmacytosis $(57.6 \%)$, paracortical expansion $(12.1 \%)$ and periadenitis $(30.3 \%)$. In the present study, AFB was positive in all the 33 cases. Two cases were diagnosed as tuberculosis on biopsies and confirmed as atypical mycobacterium

Conclusion: Lymph node biopsy is a valuable tool in the evaluation of HIV positive patient to identify the causes of lymphadenopathy.

Keywords: HIV, tuberculosis, lymph node biopsy, histological features, bacilli load
Volume 2 Issue 5 - 2017

\author{
Lakshmi Agarwal,' Manmohan Agrawal,'2 \\ Geetha V, ${ }^{3}$ Rajiv Saxena' \\ 'Department of pathology, Government medical college, India \\ ${ }^{2}$ Department of surgical oncology, Pushpadi cancer care centre, \\ India \\ ${ }^{3}$ Department of pathology, Kasturba Medical College, India
}

Correspondence: Lakshmi Agarwal, Consultant oncopathologist AP, Department of pathology, Government medical college, Pushpadi cancer care centre, India, Tel 9772464587 , Emaildrlaxmiagarwal@gmail.com

Received: October 31, 2017 | Published: December 18, 2017

\section{Introduction}

Lymphadenopathy is frequent in persons with HIV infection, occurring either as one of the earliest manifestations of infection or as a finding at any time throughout the clinical course of progression through AIDS. Tuberculosis is one of the most common causes of lymphadenopathy in HIV positive patients. The presenting complaint is same as tuberculosis in non-HIV patients. However, the histologic features of lymph node in tuberculosis vary depending on the immune status of the patients. The present study is conducted to find out these differences and its relevance in diagnosing.

\section{Materials and methods}

The histological features seen on lymph node biopsies, done on HIV positive patients who presented with lymphadenopathy, with or without other systemic manifestations over a period of three years were analyzed. Patients aged less than 14 years and inadequate samples were excluded from the study. Seventy-four lymph node biopsies were adequate and provided the material for the study. In each case, a brief and precise history was taken which included age, presenting complaints, any opportunistic infection etc. Complete blood picture and CD4+, CD8+ counts were noted wherever available. Results of Mantoux test, sputum for AFB and ESR when done were also documented. The lymph nodes biopsies were fixed in $10 \%$ formalin and were routinely processed. The histopathological evaluation of all cases was done using Haematoxylin and eosin (H\&E) stain. The number of slides studied in each case varied from 1 to 6 . Sections were stained for AFB by using Ziehl-Neelson method if $\mathrm{H} \& \mathrm{E}$ stained slides showed features suggestive of tuberculosis on light microscopy.

\section{Results}

In the current study, 33 cases (44\%) were diagnosed as tubercular lymphadenitis and were the second most common causes of lymphadenopathy after reactive hyperplasia. The presenting complaints in this group were fever $(100 \%)$ followed by cough $(84 \%)$ and weight loss $(75 \%)$. Generalized lymphadenopathy was seen in 25 cases $(75.6 \%)$ where as cervical lymphadenopathy was found in 7 cases $(21.2 \%)$. One case presented with axillary lymphadenopathy. All the patients (33) were clinically diagnosed as AIDS. A raised ESR was seen in all cases ranging from 40 to $140 \mathrm{~mm} / \mathrm{hr}$. The CD4 counts of all these patients ranged from 10-258/ $\mu 1$ with the CD4 count being $<200$ in $84.8 \%$ of cases (Table 1). Mantoux test and sputum for 
AFB were performed in 22 cases and was strongly positive in all of them. Granulomas were detected in $90.9 \%$ of the cases and were the most common and conspicuous feature. Micro abscess with granular debris without coexisting granuloma was found in 11 cases $(33.3 \%)$.

Table I HIV positive patients with tuberculous lymphadenitis: CD4 counts $(n=33)$

\begin{tabular}{lll}
\hline CD4counts/ $\boldsymbol{\mu l}$ & Number of cases & Percentage \\
\hline $0-40$ & 11 & 33.3 \\
$41-80$ & 8 & 24.2 \\
$81-120$ & 2 & 6 \\
$|2|-160$ & 7 & 21.3 \\
$|6|-200$ & 0 & 0 \\
$>200$ & 5 & 15.2 \\
\hline
\end{tabular}

\section{Discussion}

Tuberculosis (TB) was not mentioned as a manifestation of AIDS in the earlier description of the disease from USA and Europe. The association with TB was recognized in Haitians and intravenous drug abusers. ${ }^{1}$ In developing countries, it is now recognized as one of the most common opportunistic infection in seropositive patients. It is unclear whether HIV associated TB is usually a primary, reactivated or secondary exogenous infection though reactivation of latent infection is the most important cause. Whatever the source, people infected by both HIV and TB have an accelerated progression to overt tuberculosis. ${ }^{2,3}$ Cervical lymphadenopathy was the most common site of localized lymphadenopathy in the current study and this is similar to the findings of Hadadi et al..$^{4}$ In studies conducted by 'Chaisson et al. ${ }^{5} \&$ Duncanson et al. ${ }^{6}$ and coworkers ${ }^{5,6}$ it was noted that TB usually precedes the diagnosis of AIDS, presumably because TB is more virulent than other HIV associated pathogens. Their data also confirms that TB is an AIDS related opportunistic infection characterized by atypical clinical and radiological features and poor survival. Gnana et al. noted a greater prevalence of extra pulmonary and disseminated forms of TB in HIV positive individuals than in immunocompetent patients. Diagnosing extra pulmonary TB in patients with HIV is important because it is an AIDS defining illness. The most frequent forms are lymphadenitis and miliary disease. ${ }^{1,7,8}$ Lymph nodes are commonly infected by mycobacterium in patients with AIDS. ${ }^{9}$ In such patients, abscesses and purulent inflammation comprise a significant component of the histologic features of tuberculous lymphadenitis. Morphologically, in addition to the presence of polymorphs, the other criteria for the diagnosis are caseous necrosis and granulomas, of which the former is the most specific and sensitive as shown by Arora et al. ${ }^{10} \&$ Nambuya et al. ${ }^{11}$ in their study on lymph nodes morphology in HIV positive patients with TB classified the tubercular node into four categories based on cellularity and number of AFB's. These were:

I. Reactive 2+: Caseating tuberculosis + large areas of necrosis, focal granulomas and langhan's giant cells $(\mathrm{AFB}<1 / 100 \mathrm{HPF})$.

II. Reactive 1+: Same as group A, but few tubercles, epithelioid cells, langhan's giant cells(AFB1/10-100HPF).

III. Hyporeactive: Necrosis with some epithelioid cells but no giant cells (AFB1-10/HPF)

IV. Nonreactive: Areas of necrosis + nuclear debris, no epithelioid cells or giant cells(AFB $>10 / \mathrm{HPF})$.
Shobhana et al. ${ }^{12}$ in their study documented $41 \%$ of their cases as tuberculous lymphadenitis with their CD4 counts varying between 113 and 422 cells/ $\mu \mathrm{L}$. The various morphological features analyzed in cases of tuberculosis included; presence and type of granulomas, neutrophilic micro abscess, periadenitis, plasmacytosis and paracortical expansion (Table 2). The morphology of the granulomas i.e. discrete or confluent and presence or absence of caseation was also noted. In the present study, granulomas (Figure 1A) (Figure 1B) were detected in $90.9 \%$ of the cases and were the most common and conspicuous feature (Table 3). Among the 30 cases with granulomas, caseous necrosis (Figure 2) was found in 28 (93.3\%). Confluent granulomas were more commonly seen than discrete ones (Table 3 ). These findings are similar to those of other workers. ${ }^{8,9,10}$ Caseation alone was the most common specific and sensitive findings in most studies. Wannakrairot et al. ${ }^{13}$ not only found this to be the predominant feature in $74.5 \%$ of their cases but also found granulomas to be less numerous in AIDS than in non-AIDS patients. ${ }^{13,14}$ Perenboom et al. ${ }^{15}$ observed that microscopic caseation was $100 \%$ predictive of tuberculosis with a sensitivity of $69 \%$. Most of the cases of caseating (75\%) had more than $1 \mathrm{AFB} / \mathrm{hpf}$ whereas in the remaining cases $(25 \%)$, number was less than 1/100hpf. ${ }^{15}$ Granulomatous lymphadenitis without caseous necrosis was seen in 2 cases $(6.7 \%)$. These were subsequently diagnosed as tuberculosis based on AFB positivity. Similar non caseous granuloma may also be seen in opportunistic fungal infections, of which Candida, Cryptococcus neoformans, Histoplasma capsulatum have been reported in HIV positive patients. Hence, in cases with non caseating granulomas, AFB stain is very useful to identify tubercle bacilli and thereby ascertain the etiology of the granulomatous process. Nag et al found the highest AFB positivity was among the patients showing caseation necrosis plus ill-formed granuloma $(100 \%)$, followed by caseation necrosis only $(80 \%) .{ }^{9} \mathrm{He}$ also documented two cases of histoplasmosis and cryptococcosis in which well-formed epithelioid granuloma without any necrosis was present. ${ }^{9}$
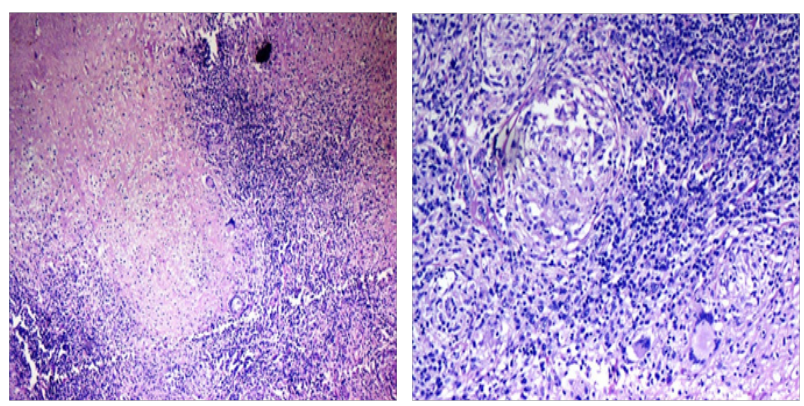

Figure I (A) Granulomas(H\&E I OX). (B) Granulomas(H\&E40X).

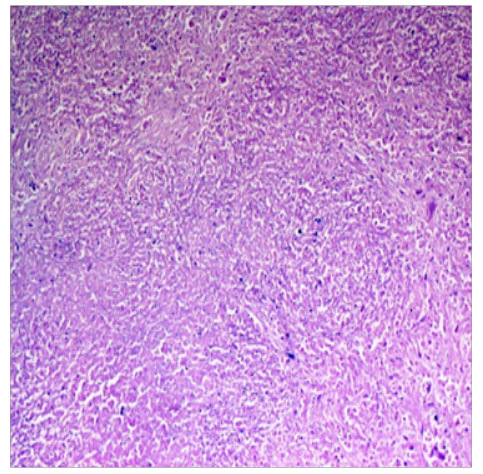

Figure 2 Caseous necrosis(H\&EIOX). 
Table 2 HIV positive patients with tuberculous lymphadenitis -Microscopic features $(n=33)$

\begin{tabular}{lll}
\hline Features & Number of cases & Percentage \\
\hline Granulomas & 30 & 90.9 \\
Neutrophilic microabscess & 11 & 33.3 \\
Periadenitis & 10 & 30.3 \\
Plasmacytosis & 19 & 57.6 \\
Paracortical expansion & 4 & 12.1 \\
AFB positivity & 33 & 100 \\
\hline
\end{tabular}

Table 3 HIV positive patients with tuberculous lymphadenitis: Type of granulomas $(n=30)$

\begin{tabular}{llll}
\hline Granulomas & Discrete & Confluent & Both \\
\hline Caseating $(n=28)$ & $9(32.1 \%)$ & $14(50 \%)$ & $5(17.9 \%)$ \\
Non caseating $(n=2)$ & 0 & $2(100 \%)$ & 0 \\
\hline
\end{tabular}

In the present study, micro abscess (Figure 3A) (Figure 3B) with granular debris without coexisting granuloma was found in 11 cases $(33.3 \%)$ (Table 1) and all showed acid fast bacilli on Ziehl-Neelsen stain. In the absence of granulomas, the identification of such micro abscesses with granular debris should therefore raise the suspicion of tuberculosis in these immunocompromised patients. An AFB can be performed to confirm the same. In the current study, tuberculosis was diagnosed in these 11 patients on the basis of AFB positivity. Ukekwe et al. ${ }^{8}$ also opined that histological demonstration of AFB by Ziehl-Neelsen stain in TB is the goal standard for a diagnosis of TB. ${ }^{8}$ Four cases (36\%) showed langhans giant cells. Priyanka Chand et al. ${ }^{14}$ found caseous necrotic material with degenerated inflammatory cells without epithelioid cell granulomas in $34.4 \% .{ }^{14}$ Wannakrairot et al. ${ }^{13}$ in their study noted that extensive nuclear debris was present in $10 \%$ of their cases, sometimes with scattered neutrophils and occasional eosinophils. ${ }^{13}$ Langerhans cells were rarely present. The necrosis was accompanied by a minimal granulomatous response that was not distinctive and was discernible only focally. The reaction was represented by scattered epithelioid histiocytes associated with marked intervening edema. Shenoy ${ }^{16} \&$ Jayaram $^{17} \&$ Kraus $^{18}$ along with their co workers have noted neutrophilic micro abscesses in approximately $30 \%$ of their cases and are in concordance with findings of the present study. ${ }^{16-18}$ In these cases, with neutrophilic micro abscesses $(n=11)$; it was noted that the bacillary load was higher in patients with lower CD4+ count (Table 4). These findings are similar to those of Nambuya et al. ${ }^{11}$ They noted that numerous bacilli were found in granular debris in their study group. It was seen in 6 of the 11 cases $(54.5 \%)$ of patients with CD4 count $<40$. Similarly, patients with low CD4 count $(<200)$ were found to have high bacilli load i.e. $72.2 \%$. The relationship of CD4+ count with presence of micro abscess and AFB in present study is in concordance with that of Nambuya et al. ${ }^{11}$ This unusual histopathology of tuberculosis in AIDS (i.e. abscesses and organisms) has been attributed to a compromised immune status and an inability to mount an adequate cellular response. Other features noted in present study were plasmacytosis $(57.6 \%)$, paracortical expansion (12.1\%) and periadenitis(30.3\%). Ngilimana et al. ${ }^{19}$ in their study documented the presence of numerous blood vessels and abundant plasmacytosis in the non-necrotic areas. ${ }^{19}$ The gold standard for a definitive diagnosis of tuberculosis rests on the demonstration of AFB on histopathology or by culture studies. In the present study,
AFB was positive in all the 33 cases. Bacilli load was categorized into four groups depending on the number of AFB's present per high power field (HPF) i.e. AFB $<1 / 100 \mathrm{HPF}$, AFB1-10/HPF, AFB $>1 / 10-100 \mathrm{hpf}$, $\mathrm{AFB}>10 / \mathrm{HPF}$ and the results are depicted in tabular form (Table 5). All these patients were clinically categorized as AIDS. None were in the ARC or AIDS at risk categories.

Table 4 HIV positive patients with tuberculous lymphadenitis: correlation between microabscess formation, CD4+ count and AFB $(n=I I)$

\begin{tabular}{lllll}
\hline CD4+inul & Microabscess & AFB<I/100HPF & $\begin{array}{l}\text { AFB } \\
\text { I-I0/ } \\
\text { HPF }\end{array}$ & $\begin{array}{l}\text { AFB }>10 / \\
\text { HPF }\end{array}$ \\
\hline$<40$ & 6 & NIL & 3 & 3 \\
$120-160$ & 2 & NIL & 1 & 1 \\
$>200$ & 3 & 2 & 1 & NIL \\
\hline
\end{tabular}

Table 5 HIV positive patients with tuberculous lymphadenitis: Evaluation of bacillary load $(n=33)$

\begin{tabular}{lllll}
\hline $\begin{array}{l}\text { Bacilli } \\
\text { load }\end{array}$ & AFB<I/I00hpf & $\begin{array}{l}\text { AFBI-I0/ } \\
\text { hpf }\end{array}$ & $\begin{array}{l}\text { AFB }>1 / 10- \\
\text { I00hpf }\end{array}$ & AFB>I0/hpf \\
$\begin{array}{l}\text { Number } \\
\text { of cases }\end{array}$ & 10(30.3\%) & I3(39.3\%) & $2(6 \%)$ & $8(24.4 \%)$ \\
\hline
\end{tabular}
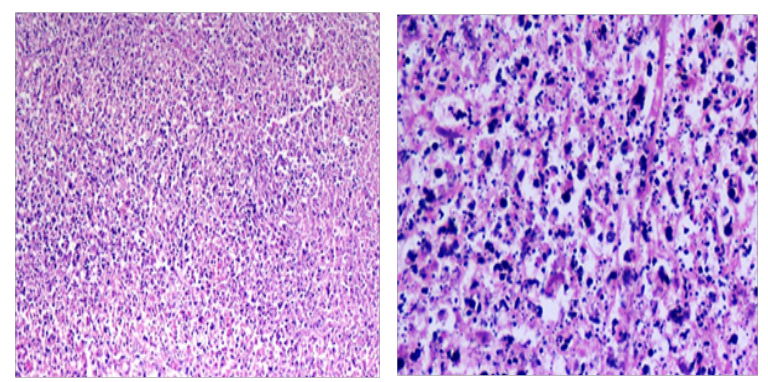

Figure 3 (A) Neutrophilic microabscess with granular debris(H\&E IOX). (B) Neutrophilic microabscess with granular debris (H\&E 40X).

Nambuya et al. ${ }^{11}$ in their study observed that the number of AFB's tended to correlate with AIDS status. These patients were more likely to have abundant AFB than those with AIDS related complex and AIDS risk patients. Further, no AIDS patients with a positive mycobacterial culture had a negative AFB stain. Mycobacterium avium and mycobacterium intracellulare are two closely related, non-chromogenic mycobacteria that are grouped together to form the Mycobacterium avium intracellular complex (MAC). These are the second most common mycobacteria isolated from patients with AIDS. Unlike TB, MAC disease occurs later in the course of AIDS. The histopathology of MAC infected tissues reveals non-necrotizing granulomas which are often poorly formed and associated with clusters of foamy macrophages. Klatt et al. ${ }^{20}$ studied pathologic findings of disseminated MAI infection in 12 patients with AIDS. In every patient, the distinctive microscopic features on H\&E staining were poorly defined granulomas consisting of pale blue striated histiocytes filled with mycobacteria. Well-formed granulomas with fibrosis, necrosis and epithelioid histiocytes were present in less than one third of the cases. It occurs as a late complication of HIV infection, often with $\mathrm{CD} 4+$ count less than $50 / \mu 1$. In the present study, two cases were diagnosed as tuberculosis on biopsies and confirmed as atypical mycobacterium by culture. However neutrophilic micro abscesses 
were also found in 9 cases of lymphadenitis due to Mycobacterium tuberculosis. Thus, these findings of neutrophilic micro abscess are not specific for atypical Mycobacteria. However, presence of such micro abscesses should raise the suspicion of atypical mycobacterial infection and culture should be requested for definite diagnosis. Kraus et al had opined that culture should be done in presence of micro abscess and ill-defined granulomas to rule out atypical mycobacteria. ${ }^{18}$

The various clinical and microscopic features in case of high and low bacillary load were compared and are depicted in a tabular form in Table 6. No statistically significant difference was noted in the

Table 6 Tuberculous lymph nodes: Clinical and morphological features in high and low bacillary load groups

\begin{tabular}{|c|c|c|}
\hline Features & High bacillary loadAFB $>10 / \mathrm{Hpf}(\mathrm{N}=8)$ & Low bacillary loadAFB $<1 / 100 h p f(N=10)$ \\
\hline Common clinical features & Fever and cough & Fever and cough \\
\hline \multicolumn{3}{|l|}{ Lymphadenopathy: } \\
\hline Generalized & $50 \%$ & $80 \%$ \\
\hline Localized & $50 \%$ & $20 \%$ \\
\hline ESR in mm/hour (mean) & 110 & 80 \\
\hline Mantoux Test (N=4 In Each Group) & Positive in one case & Positive in one case \\
\hline \multicolumn{3}{|l|}{ CD4+count: } \\
\hline$<50 / \mu l$ & $50 \%$ cases & $20 \%$ cases \\
\hline$>50-<200 / \mu \mathrm{l}$ & $50 \%$ cases & $80 \%$ cases \\
\hline Caseation & $87.50 \%$ & $90 \%$ \\
\hline Neutrophilic microabscess & $62.50 \%$ & $20 \%$ \\
\hline \multicolumn{3}{|l|}{ Granulomas: } \\
\hline Confluent & 5 cases & 6 cases \\
\hline Discreet & 3 cases & Nil \\
\hline Absent & Nil & one case \\
\hline Both(discrete and confluent) & Nil & 3 cases \\
\hline Langhans giant cells & I case(|2.5\%) & $4 \operatorname{coses}(40 \%)$ \\
\hline
\end{tabular}

\section{Conclusion}

Caseous necrosis was a distinctive feature even in the absence of granulomas or in the presence of poorly formed ones in case of tuberculosis. Neutrophilic micro abscess with granular debris without co existing granulomas had higher bacillary load. This stresses the need for routine AFB staining in all such cases. Hence lymph node biopsy along with AFB staining is a valuable tool in the evaluation of HIV positive patient to identify the cause of lymphadenopathy and to confirm the diagnosis of tuberculosis. ${ }^{21}$

\section{Acknowledgements}

None.

\section{Conflict of interest}

The author declares no conflict of interest.

\section{References}

1. Sunderam G, Mc Donald RJ, Maniatis T, et al. Tuberculosis as a manifestation of the acquired immunodeficiency syndrome (AIDS). JAMA. 1986;256(3):362-366. two groups with respect to clinical presentation and morphological features. A higher rate of acid fast bacilli positivity $(62.5 \%)$ was found in cases with neutrophilic micro abscess, most of which did not show discreet granulomas. Langhans giant cell was found in only 1 case $(12.5 \%)$. Therefore, in the absence of Ziehl-Neelsen staining, these cases would have been dismissed as acute necrotizing lymphadenitis. Though marked difference in percentage was observed between the two groups in relation to neutrophilic micro abscess $(62.5 \%$ and $20 \%$ respectively), it was not statistically significant. This discrepancy may be attributed to the small sample size available for analysis.
2. Pitchenik AE, Fertel D, Bloch AB. Mycobacterial disease: Epidemiology, diagnosis, treatment and prevention. Clin Chest Med.1988;9(3):425441.

3. Bem C, Patil PS, Bharucha H, et al. Importance of human immunodeficiency virus-associated lymphadenopathy and tuberculous lymphadenitis in patients undergoing lymph node biopsy in Zambia. BrJ Surg. 1996;83(1):75-78.

4. Hadadi A, Jafari S, Jebeli ZH, et al. Frequency and etiology of lymphadenopathy in Iranian HIV/AIDS patients. Asian Pac J Trop Biomed. 2014;4(Suppl 1):S171-176.

5. Chaisson RE, Schecter GF, Theuer CP, et al. Tuberculosis in patients with acquired imuno deficiency syndrome. Clinical features, response to therapy and survival. Am rev respire Dis. 1987;136(3):570-574.

6. Duncanson FP, Hewlett D Jr, Maayan S, et al. Mycobacterium tuberculosis infection in acquired immunodeficiency syndrome: A review of 14 patients. Tubercle. 1986;67(4):295-303.

7. Kamana NK, Wanchu A, Sachdeva RK, et al. Tuberculosis is the leading cause of lymphadenopathy in HIV-infected persons in India: Results of a fine-needle aspiration analysis. Scand J Infect Dis. 2010;42(11-12):827830 . 
8. Ukekwe F, Olusina DB, Banjo A, et al. Tuberculous lymphadenitis in South-Eastern Nigeria; A 15 years histopathologic review (2000-2014). Ann Med Health Sci Res. 2016;6(1):44-49.

9. Nag D, Dey S, Nandi A, et al. Etiological study of lymphadenopathy in HIV-infected patients in a tertiary care hospital. J Cytol. 2016;33(2):6670

10. Arora B, Arora DR. Fine needle aspiration cytology in tuberculous lymphadenitis. Ind J Med Res. 1991;1991:189-192.

11. Nambuya A, Sewankambo N, Mugerwa J, et al. Tuberculous lymphadenitis associated with human immune deficiency virus in Uganda. J Clin Pathol. 1988;41(1):93-96.

12. Shobhana A, Guha SK, Mitra K, et al. People living with HIV infection/ AIDS-A study of lymph node FNAC and CD4 count. Ind J Med Microbiol. 2002;22(2):99-101.

13. Wannakrairot P, Leong MT, Anthonu S. The morphological spectrum of lymphadenopathy in HIV infected patients. Pathology. 2007;39(2):223227.

14. Chand P, Dogra R, Chauhan N, et al. Cytopathological Pattern of Tubercular Lymphadenopathy on FNAC: Analysis of 550 Consecutive Cases. J Clin Diagn Res. 2014;8(9):FC16-C19.
15. Perenboom RM, Richter C, Swai AB, et al. Diagnosis of tuberculous lymphadenitis in an area of HIV infection and limited diagnostic facilities. Trop Geogr Med. 1994;46(5):288-292.

16. Shenoy R, Kapadi SN, Pai KP, et al. Fine Needle Aspiration diagnosis in HIV related lymphadenopathy in Mangalore, India. Acta Cytol. 2002;46(1):35-39.

17. Jayaram G, Chew MT. FNAC of lymph node in HIV infected individuals. Acta Cytol. 2000;44:960-966.

18. Kraus M, Benharroch D, Kaplan D, et al. Mycobacterial cervical lymphadenitis: The histological features of non-tuberculous mycobacterial infection. Histopathology. 1999;35(6):534-538.

19. Ngilimana PJ, Metz T, Munyantore S, et al. Lymph node tuberculosis in HIV 1 seropositive patients in central Africa. A characteristic histopathologic picture. Ann Pathol. 1995;15(1):38-44.

20. Klatt EC, Jensen DF, Meyer PR. Pathology of Mycobacterium avium-intracellulare infection in acquired immunodeficiency syndrome. Hum Pathol. 1987;18(7):709-714

21. Pinder SE, Colbille A. Mycobacterial cervical lymphadenitis in children; can histological assessment help differentiate infection caused by non-tuberculous mycobacteria from mycobacterium tuberculosis. Histopathology. 1993;22(1):59-64. 